\title{
Columnar Cell Variant of Papillary Thyroid Carcinoma: A Clinicopathologic Analystis
}

\author{
Zhang Gang ${ }^{1}$, Zhao Xing ${ }^{2}$, Sun Jirui ${ }^{3}$, Zhang Jinku ${ }^{3}$, Zhang Jinzhuo ${ }^{4, *}$ \\ ${ }^{1}$ Department of Surgical Oncology, The First Central Hospital of Baoding, Baoding, China \\ ${ }^{2}$ Department of Pathology, Chengde Medical College Affiliated Hospital, Chengde, China \\ ${ }^{3}$ Department of Pathology, The First Central Hospital of Baoding, Baoding, China \\ ${ }^{4}$ Department of Digestive System, The First Central Hospital of Baoding, Baoding, China \\ Email address: \\ 86781038@qq.com (Zhang Gang), 435444923@qq.com (Zhang Jinzhuo) \\ ${ }^{*}$ Corresponding author
}

\section{To cite this article:}

Zhang Gang, Zhao Xing, Sun Jirui, Zhang Jinku, Zhang Jinzhuo. Columnar Cell Variant of Papillary Thyroid Carcinoma: A Clinicopathologic Analystisa. European Journal of Clinical and Biomedical Sciences. Vol. 5, No. 1, 2019, pp. 16-20. doi: 10.11648/j.ejcbs.20190501.14

Received: October 14, 2018; Accepted: January 21, 2019; Published: May 8, 2018

\begin{abstract}
Background: Papillary Thyroid Carcinoma (PTC) is the Most common thyroid malignancy disease, and it is more common in females. It happened in any age. Mean age at diagnosis is approximately 40 years old. There is history of neck irradiation in $5-10 \%$ patients. And non-neoplastic gland may show nuclear aberrations as a result. There is increased incidence in Hashimoto's thyroiditis, but not clear whether there is an increased incidence in Graves' disease. Columnar cell variant of papillary thyroid carcinoma is the rare subtype of PTC, which is reportedly about $0.17 \%$. CCV-PTC was reported in 1986 by Evans for the first time. It is different from the typical papillary carcinoma, not only in morphology but also in biological behavior, and is more aggressive than typical papillary carcinoma. Objective: To study the clinical-pathological links and features of papillary thyroid carcinoma (Columnar cell variant) (CCV-PTC). Methods: 4 cases of CCV-PTC from Chengde medical college affiliated hospital were retrospectively analyzed, to observe its clinical features, histological, immunophenotype, and metastasis. And the literature of CCV-PTC were reviewed. Results: Out of the 4 patients, two male patients of 70 and 53 years old, two female patients of 64 and 68 years old. Microscopically, tumor cells arranged in multistage papillary structure, with pseudostratified columnar epithelium, same to gastrointestinal and lung metastatic carcinoma. IHC: Ki-67(5-15\%), calcitonin(-), TG(+), ck19(-), TTF-1(+), $\mathrm{CgA}(-), \operatorname{SyN}(-), \mathrm{ck}(+/-)$. Conclusion: CCV-PTC is a special subtype of PTC with a more aggressive biological behavior, particularly among older people, Immunohistochemical method is helpful for the diagnosis.
\end{abstract}

Keywords: Papillary Thyroid Carcinoma, Columnar Cell Variant, Pathological Feature

\section{Introduction}

Papillary thyroid carcinoma is the most common thyroid cancer, about $60 \%-70 \%$, and often encountered in children and adolescents, especially young women [1-3]. Age has a significant impact on prognosis, which is good in young patients and poor in old patients. The histologic subtypes of papillary thyroid carcinoma included follicular variant, diffuse sclerosing variant, columnar cell variant, tall cell variant, oxyphilic cell variant, Warthin's-like variant, papillary thyroid carcinoma with nodular fasciitis-like stroma., cribriform papillary thyroid carcinoma, and radiation-induced pediatric thyroid cancer. CCV-PTC is a rare type of papillary thyroid carcinoma of high malignancy. 4 cases of CCV-PTC were collected, to discuss the histopathological characteristics, genetics, and prognosis.

\section{Materials and Methods}

\subsection{Clinical Data}

Case 1 Female, 64 years old, the tumor was located in the right leaf of the thyroid, about $3.0 \mathrm{~cm} \times 3.0 \mathrm{~cm}$. Boundary clear, smooth surface, without tenderness were the mainly clinical symptoms. B-ultrasonography showed several hypoechoic nodules, weak echo nodular and hybrid echo-mass in thyroid parenchyma of double sides. The largest nodule was 
$25.3 \mathrm{~mm} \times 16.8 \mathrm{~mm} \times 24.1 \mathrm{~mm}$ in right side, with clear boundary and relatively regular shape. CDFI: color flow was found surrounding the nodule.

Case 2 Male, 70 years old, with III degree swelling of thyroid. Multiple nodules was in left side, out of which the largest one was $8.0 \mathrm{~cm} \times 15.0 \mathrm{~cm}$. Boundary clear, smooth surface, without tenderness were the mainly clinical symptoms. No noise of blood vesselsis heard. Palpable cervical lymph node, proptosis and hands shaking were not found. CT showed slightly high density shade under sternocleidomastoid muscle of the left, about $5.5 \times 6.3 \times 10 \mathrm{~cm}$, heterogeneous enhancement and punctate calcifications.

Case 3 Male, 58 years old, the tumor was located in the right leaf of the thyroid, about $6.5 \mathrm{~cm} \times 5.5 \mathrm{~cm}$. Boundary clear, smooth surface, without tenderness were the mainly clinical symptoms. B-ultrasonography showed several medially echoic-mass in thyroid parenchyma of left sides, with clear boundary and relatively regular shape. Medially echoic-mass,about $60.0 \mathrm{~mm} \times 28.5 \mathrm{~mm} \times 63.6 \mathrm{~mm}$, was found in supraclavicular fossa.

Case 4 Female, 68 years old, $10 \mathrm{~cm}$ neoplasm was found in the neck, with unclearly boundary and very tender. CT showed high density shade in the soft tissue of front clavicle.

\subsection{Methods}

4 cases of specimens are fixed in the neutral formalin liquid for 12 hours. The samples were taken for routine histopathological observation. IHC was used to be diagnosed definitively. Ki-67, calcitonin, TG, ck19, TTF-1, CgA, SyN and CK were from Jinqiao Company in Beijing, and compared with the positive control group and negative control group.

\section{Results}

\subsection{Macroscopic}

Case 1 Broken tissue, about $1.5 \times 1 \times 0.5 \mathrm{~cm}$, dusty red, Calcifications was seen in part area. Case 2 A gray-red nodular mass, about $10 \times 5 \times 4 \mathrm{~cm}$, with intact capsule. Case 3 Nodule, about $6.5 \times 5 \times 5 \mathrm{~cm}$, with clear boundary, gray-red, firm. Case 4 Nodule, about $13 \times 7 \times 3.5 \mathrm{~cm}$. gray-red.

\subsection{Microscopically}

Tumor cells arranged in multistage papillary structure, with pseudostratified columnar epithelium, same to gastrointestinal and lung metastatic carcinoma. (Figure 1-4).

\subsection{Immunohistochemistry}

TG and TTF-1 were positive in tumor cells of CCV-PTC, while Calcitonin, CK19, CgA, SyN were negative. CK was weakly positive. $\mathrm{Ki}-67$ (5-15\%). (Figure 5-10).

\subsection{Genetics}

Gene rearrangements of the PTC was RET/PTC, include RET/PTC1, RET/PTC2 and RET/PTC3, which were associated with the histological subtypes, such as RET/PTC1 in occult thyroid cancer and PTC, RET/PTC3 in tall cell variant. Mutated BRAF was found in CCV-PTC [4].

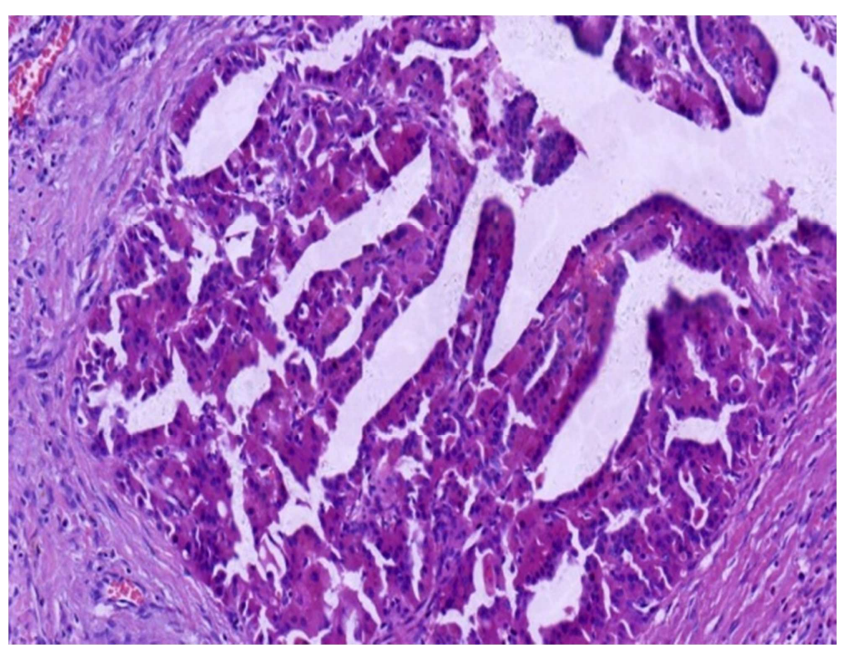

Figure 1. Tumor cells arranged in multistage papillary structure.

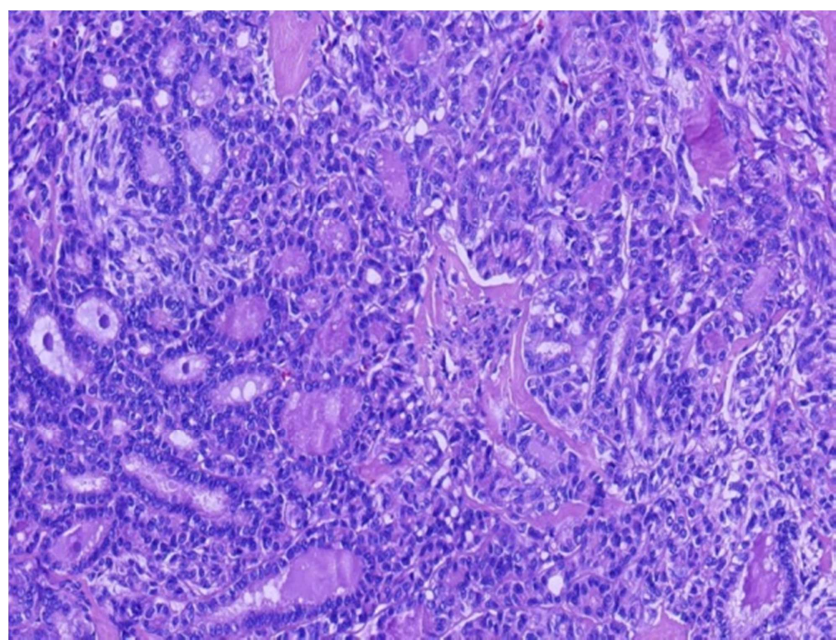

Figure 2. Invasive tumor cells HE 200X.

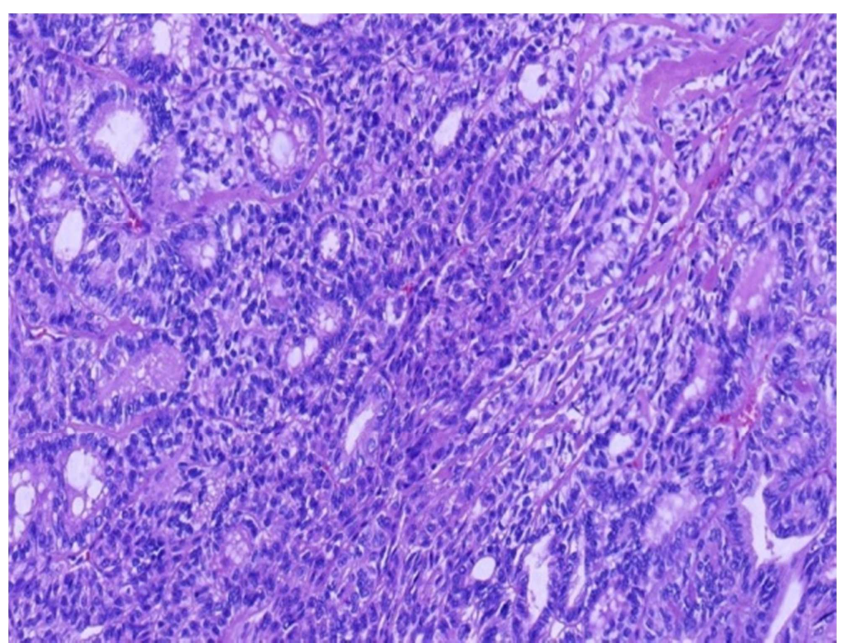

Figure 3. nuclear vacuolization in tumor cells. 


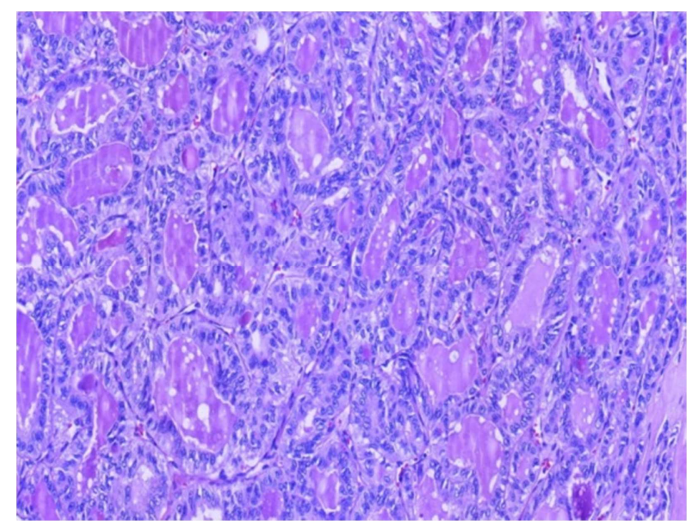

Figure 4. pseudostratified columnar epithelium.

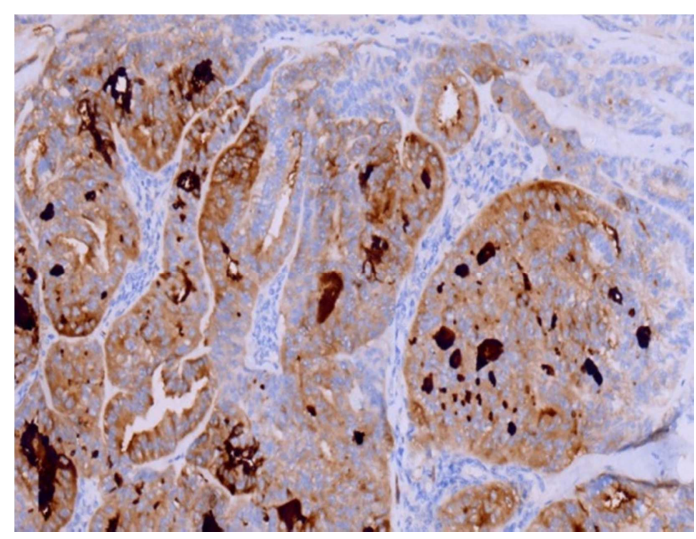

Figure 5. The positive expression of TG in tumor cells 200X.

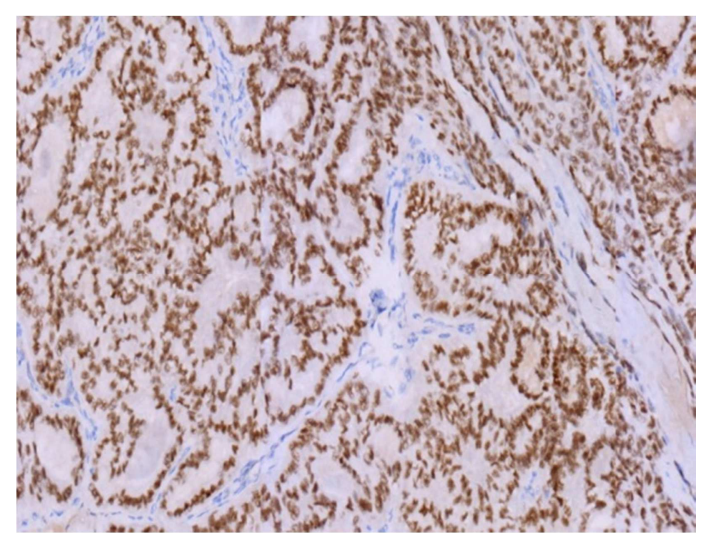

Figure 6. The positive expression of TTF-1 in tumor cells 200X.

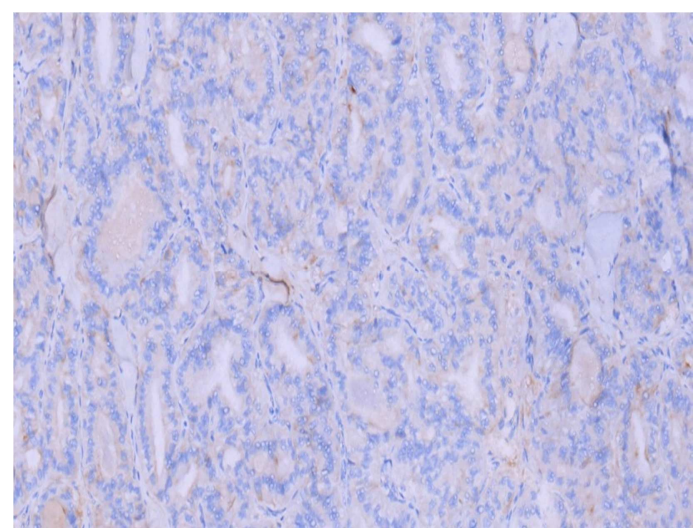

Figure 7. The negative expression of CK19 in tumor cells 200X.

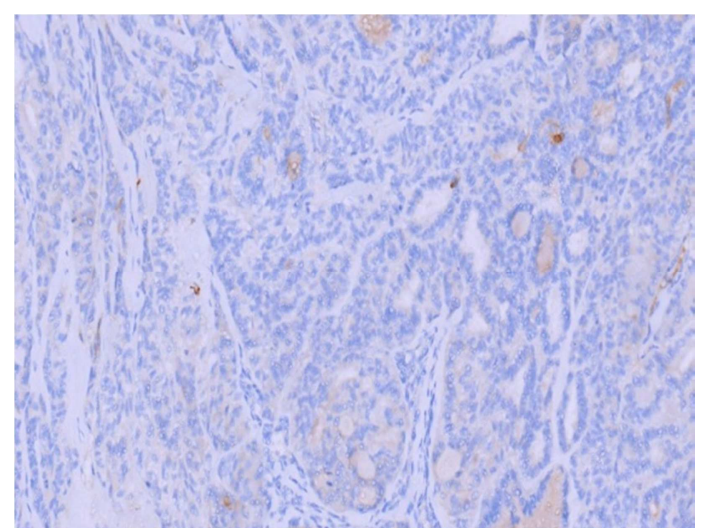

Figure 8. The negative expression of Calcitonin in tumor cells $200 X$.

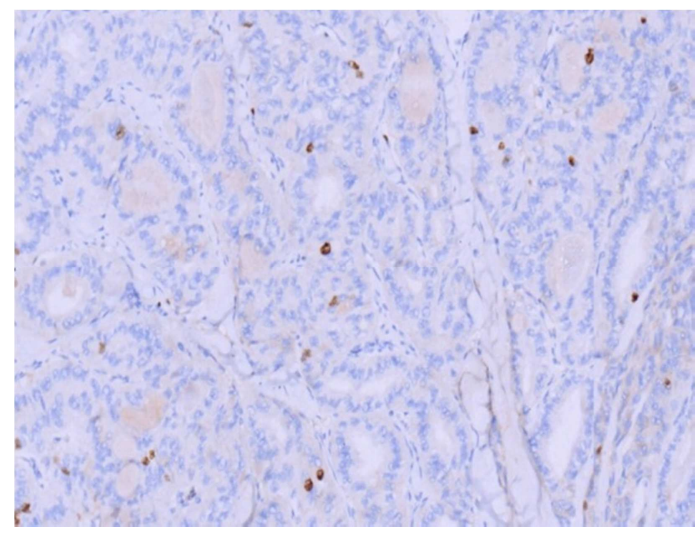

Figure 9. The expression of Ki-67 in tumor cells 200x.

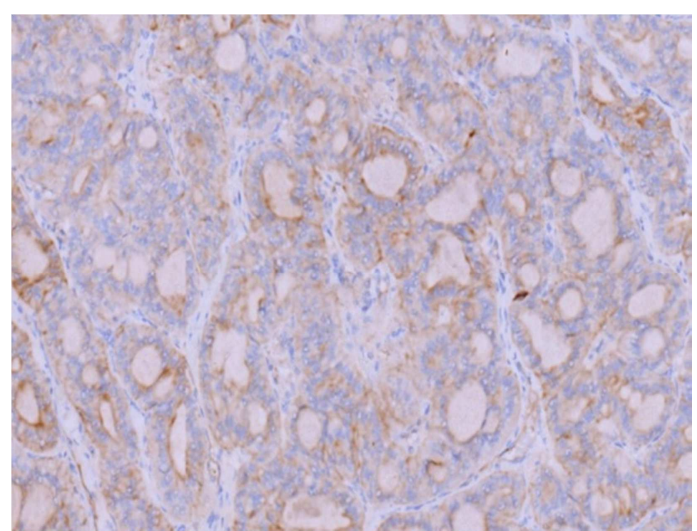

Figure 10. The weakly positive expression of CK in tumor cells 200x.

\section{Discussion}

Papillary thyroid carcinoma (PTC) is the most common malignancy in the thyroid gland, which accounts for $90 \%$ of all thyroid cancers. The variant of papillary carcinoma of the thyroid represents an unusual neoplasm whose clinicopathological features and biological behavior have not been thoroughly characterized.Columnar cell variant of papillary thyroid carcinoma is the rare subtype of PTC, which is reportedly about $0.17 \%$ [5]. The revised American Thyroid Association guidelines recently categorized the PTC variants according to their biological behavior as described in the literature, and CCV-PTC was classified as the aggressive type. 
CCV-PTC was reported in 1986 by Evans for the first time [6]. It is different from the typical papillary carcinoma, not only in morphology but also in biological behavior, and is more aggressive than typical papillary carcinoma.

\subsection{Clinical Symptoms}

CCV-PTC is seen in older women, who with a worse prognosis than young women [7]. The Surveillance of 765 cases of CCV-PTC was from 1988 to 2013 by C Jiang [8]. The study showed, Compared with PTC, CCV tumors tended to be larger, with a higher incidence rate among males and in patients $\geq 65$ years of age. CCV was associated with higher rates of extrathyroidal extension, multifocality, lymph node examinations, and lymph node and distant metastases $(\mathrm{p}<0.0001)$. Significant differences were found in 10 -year overall survival $(97.14 \%$ vs $89.15 \%, \quad \mathrm{p}<0.0001)$ and disease-specific survival $(99.08 \%$ vs $93.07 \%, \mathrm{p}<0.0001)$ between PTC and CCV. Clinical Differential Diagnosis 1. Thyroid Adenoma Thyroid adenoma is often seen in young women. Boundary clear, smooth surface, without tenderness are the mainly clinical symptoms. The neck ultrasound is helpful to establish the diagnosis. 2. Nodular Goiter Multiple nodules or solitary nodule in thyroid tissue. 3. Thyroid Cancer tends to have more advanced tumors than others with swollen lymph glands, hoarse voice and difficulty swallowing.

\subsection{Macroscopic}

There is a certain relationship between macroscopic and aggressive, such as tumor size, capsule and tumor boundary. According to the macroscopic, CCV-PTC is divided Into inert columnar cells papillary carcinom (tumor diameter smaller, no capsular invasion, boundary clear). Conversely, if the tumor diameter, no envelope is more aggressive.

\subsection{Microscopically}

Among the aggressive variants of PTC, CCV-PTC is the most misdiagnosed and underrecognized entity.

On histology, it is defined by papillae or gland - like structures lined by columnar cells displaying prominent nuclear stratification.

Tumor cells arranged in multistage papillary structure, with pseudostratified columnar epithelium, round nucleus, cell hyperplasia and cytoplasmic spaces, same to gastrointestinal and lung metastatic carcinoma. Spindle cells and follicle suggested poor prognosis. It was difficult to different CCV-PTC from tall cell variant. It was also arranged in pseudostratified columnar epithelium in tall cell variant, but acidophilic granular cytoplasm, and nucleus on the basal part of cells.

\subsection{The Role of Immunohistochemical in the Differential Diagnosis of $C C V-P T C$}

TG and TTF-1 were positive in tumor cells of CCV-PTC, which negative in gastrointestinal and lung metastatic carcinoma. CEA, EMA and CK19 were negative in tumor cells of CCV-PTC, which can different from tall cell variant(EMA,CK19,CD115 all positive).

\subsection{Genetics}

The mutation rate of BRAF gene,localed in V600E, was high in CCV-PTC, about 33\%[2]. There were 3 cases of CCV-PTC found BRAF mutation out of 9 cases, 2 cases with clinical invasive. IHC showed that, the expression of cyclin $\mathrm{D} 1$ and $\mathrm{Ki}-67$, in the nucleus, were higher, which P53 weakly positive in in invasive and inert $\mathrm{CCV}-\mathrm{PTC}$. The expression of $\beta$-catenin and $\mathrm{Bcl}-2$ were low. The expression of ER, PR in both invasive and inert CCV-PTC were high, and had nothing to do with age, gender. The expression of CDX2 was about $55 \%$, while low expression in other types of thyroid cancer. $[9,10]$.

\subsection{Survival Analysis}

A study [5] including 48 patients of CCV-PTC showed that, 20 cases were clinically indolent, and in 23 cases the tumors were considered as aggressive. Of the cases with clinical follow-up, almost all the patients with indolent tumors (18 out of 19) were alive or free from the disease for 9 months to 22 years after diagnosis. Of the 20 patients with aggressive tumors, 13 died from disease approximately $7-$ 126 months after diagnosis. Also, the extrathyroidal extension of clinically indolent CCV-PTC were not reported while most of clinically aggressive CCV-PTC showed extrathyroidal extension, accounting for $67-100 \%$ [11]. In the study of Cho, JH Shin [7], six cases of CCV-PTC were identified, the four indolent tumors in study were small (mean size: $1.2 \mathrm{~cm}$ ), encapsulated, confined to the thyroid gland, and present in younger individuals (range: 27-34 years). Recurrence was not observed in the female patients during the clinical follow-up. Most individuals in the indolent group had nodules with a smooth margin based on US. On the other hand, two patients had aggressive tumors that were larger $(1.8 \mathrm{~cm}$ and $6.0 \mathrm{~cm})$ than the indolent tumors, extrathyroidal extension, metastasis to LNs and distant organs, affected older patients (55 years and 70 years) and died of their disease, 4 years after diagnosis.

\section{Conclusion}

The prognosis of papillary thyroid carcinoma is good. $10-$ year survival rate more is than $90 \%$, especially in young patients (98\%) [12-17]. The factors affecting the prognosis included vascular invasion, nuclear atypia, tumor size and age. The prognosis of CCV-PTC is worse, because of the local invasion, lymph node metastasis and distant metastasis [18, 19]. It should be highly valued on the realization and diagnosis of CCV - PTC, to guide clinical treatment.

\section{References}

[1] Bongiovanni M, Mermod M, Canberk S, et al.Columnar cell variant of papillary thyroid carcinoma:Cytomorphological characteristics of 11 cases with histological correlation and literature review. Cancer Cytopathol, 2017, 125 (6): 389-397. 
[2] Song E, Jeon MJ, Oh HS, et al. Do aggressive variants of papillary thyroid carcinoma have worse clinical outcome than classic papillary thyroid carcinoma? Eur J Endocrinol. 2018, 179 (3): $135-142$.

[3] Ito Y, Miyauchi A, Kihara M, et al.Overall Survival of Papillary Thyroid Carcinoma Patients: A Single-Insititution Long-Term Follow-Up of 5879 Patients. World J Surg. 2018, 42 (3): 615-622.

[4] Marie Hlaváčková.Columnar cell variant of papillary thyroid carcinoma. Endocrine Abstracts, 2017, 49: 1438.

[5] Chen JH1, Faquin WC, Lloyd RV, et al. Clinicopathological and molecular characterization of nine cases of columnar cell variant of papillary thyroid carcinoma [J]. Mod Patho, 2011, 24 (5): 739-749

[6] Evans HL. Columnar-cell carcinoma of the thyroid: a report of two cases of an aggressive variant of thyroid carcinoma [J]. Am J Clin Pathol, 1986, 85 (1): 77 - 80.

[7] J Cho, JH Shin, SY Hahn, etal. Columnar Cell Variant of Papillary Thyroid Carcinoma: Ultrasonographic and Clinical Differentiation between the Indolent and Aggressive Types.

[8] Korean Journal of Radiology, 2018, 19(5): 1000. [8] C Jiang, T Cheng, X Zheng, etal. Clinical behaviors of rare variants of papillary thyroid carcinona are associated with survival: a population-level analysis. Cancer Management and Research, 2018, 10: 465-472.

[9] Rottuntikarn W, Wangsiricharoen S, Rangdaeng S. Cytomorphology and immunocytochemistry of columnar cell variant of papillary thyroid carcinoma [J]. Cytopathology, 2017, 28 (4): 338-341.

[10] Enriquez M L, Baloch Z W, Montone K T, et al. CDX2 expression in columnar cell variant of papillary thyroid carcinoma [J]. American Journal of Clinical Pathology, 2012, 137 (5): 722 .
[11] Sujoy V, Pinto A, Nosś V. Columnar cell variant of papillary thyroid carcinoma: a study of 10 cases with emphasis on CDX2 expression. Thyroid. 2013; 23: 714-71

[12] Suh S, Kim Y H, Goh T S, et al. mRNA Expression of SLC5A5 and SLC2A Family Genes in Papillary Thyroid Cancer: An Analysis of The Cancer Genome Atlas. [J]. Yonsei Medical Journal, 2018, 59 (6): 746.

[13] Kim H I, Kim T H, Choe J H, et al. Surgeon volume and prognosis of patients with advanced papillary thyroid cancer and lateral nodal metastasis [J]. British Journal of Surgery, 2018, 105 (3): 270.

[14] Liu R, Bishop J, Zhu G, et al. Mortality Risk Stratification by Combining BRAF V600E and TERT Promoter Mutations in Papillary Thyroid Cancer: Genetic Duet of BRAF and TERT Promoter Mutations in Thyroid Cancer Mortality. [J]. Jama Oncology, 2016, 3288.

[15] Yin D, Han Y, Zhang Y, et al. Clinicopathologic and neck metastasis features of multifocal papillary thyroid cancer $[\mathrm{J}]$. Chinese Journal of General Surgery, 2017 (5): 556-559.

[16] Liao T, Wen D, Ma B, et al. Yes-associated protein 1 promotes papillary thyroid cancer cell proliferation by activating the ERK/MAPK signaling pathway [J]. Oncotarget, 2017, 8 (7): 11719-11728.

[17] Liang J, Cai W, Feng D, et al. Genetic landscape of papillary thyroid carcinoma in the Chinese population [J]. Journal of Pathology, 2017, 244 (2): 215-226.

[18] Jayaram G. Cytology of columnar-cell variant of papillary thyroid carcinoma. [J]. Diagnostic Cytopathology, 2015, 22 (4): 227-229.

[19] Nath M C, Erickson L A. Aggressive Variants of Papillary Thyroid Carcinoma: Hobnail, Tall Cell, Columnar, and Solid. [J]. Advances in Anatomic Pathology, 2018, 25 (3): 1. 